\title{
Evaluation of Retinal Nerve Fibre Layer Thickness in Black Glaucoma Suspects
}

\author{
CG Nwokocha ${ }^{1}$ and CS Ejimadu ${ }^{2 *}$ \\ ${ }^{1}$ Department of Ophthalmology, Rivers State University Teaching Hospital, Nigeria \\ ${ }^{2}$ Department of Ophthalmology, University of Port Harcourt Teaching Hospital, Nigeria
}

*Corresponding author: Ejimadu Chibuike Sydney, Department of Ophthalmology,

Received Date: June 06, 2019

University of Port Harcourt Teaching Hospital, Nigeria.

Published Date: June 12, 2019

\begin{abstract}
Background: There is a need to evaluate glaucoma suspects to ascertain those who actually have the disease and commence treatment to avoid progression to blindness and monitor closely those with a tendency to progress to glaucoma. It is also necessary to rule out those without glaucoma and save them from the psychological, emotional and financial burden of being labeled a glaucoma patient. Therefore, analyzing the retinal nerve fibre layer thickness will be of great importance in achieving this.
\end{abstract}

Aim: To evaluate the retinal nerve fiber layer thickness in black glaucoma suspects.

Method: This is a non-intervention observational hospital-based study using consecutive allocation of glaucoma suspects as they presented to the glaucoma clinic between October 2nd, 2012 and January 31st, 2013, Medical history was recorded, and comprehensive ocular examination done on each of the subjects. Ocular examination included visual acuity, visual field, tonometry and ophthalmoscopy. Instruments used during the research were Pen torch for examination of the external structures of the eyes, Keeler ophthalmoscopes for fundus examination, Snellen's charts both literate and illiterate charts for visual acuity assessment, Reichert AT 555 Auto non-contact tonometer for measurement of the intra-ocular pressure. Observations were done using the Optical Coherence Tomography machine with minimum normal RNFL thickness value set at $93.46 \mu \mathrm{m}$. Data was analyzed using the statistical package EPI info version 6.04d, a software package designed by the Centers for Disease Control and Prevention (CDC), USA in 2001.

Results: A total of 120 participants comprising 60 males and 60 females were examined with a mean age of $42.8 \pm 13.79$; the age range was 19 to 75 years. The retinal nerve fiber layer was noticed to be thickest in the inferior quadrant, followed by the superior, nasal and temporal quadrants. The RNFL thickness decreased with increasing age $(p<0.05 ; r=-0.38)$. A total of $30.4 \%$ of the eyes $(n=73 / 240)$ had their average RNFL thickness lesser than $93.46 \mu \mathrm{m}$

Conclusion: The ISNT rule was obeyed in this study as the distribution of the peripapillary RNFL thickness in the various quadrants showed that the inferior quadrant had the thickest RNFL, followed by the superior, nasal and temporal quadrant. Increasing age was associated with a decrease of RNFL thickness which was statistically significant. Minority of the subjects had their average RNFL thickness lesser than the normal value.

Keywords: Retinal; Nerve; Fibre; Layer; Thickness; Glaucoma; Suspect

\section{Introduction}

Glaucoma is described as a group of diseases that have in common a characteristic optic neuropathy with associated visual function loss. Although elevated intraocular pressure (IOP) is one of the primary risk factors, its presence or absence does not have a role in the definition of the disease [1]. It is the third leading cause of blindness in the world. The prevalence of glaucoma varies slightly worldwide. The highest prevalence (4.2\%) was reported in Africa and this is likely due to the high prevalence of primary open angle glaucoma among blacks [2].
The prevalence of glaucoma blindness is increasing in most African countries as a result of the increasing life expectancy [3]. Available data suggests that age specific glaucoma prevalence in population surveys in Nigeria is about $6.5 \%$ in people over 40 years [4], given that $20 \%$ of over 150 million population are 40 years and above[5], it then implies that there are approximately 2 million people over 40 years with glaucoma in Nigeria. Of these, $90 \%$ (1.8 million) individuals are undiagnosed and there is considerable visual dysfunction at time of diagnosis.4 Published works in 
Nigeria shows that glaucoma is the second commonest cause of blindness being responsible for between $16.7 \%$ and $43.3 \%$ of cases of blindness [6-9]. Glaucoma studies done in Rivers State revealed a prevalence of $7.95 \%$ and blindness from glaucoma accounted for $20.8 \%$ of blindness in the region [10].

A glaucoma suspect is defined as an adult who has one of the following findings in at least one eye: an optic nerve or nerve fiber layer defect suggestive of glaucoma like enlarged cup-disc ratio, asymmetric cup-disc ratio, notching or narrowing of the neuro-retinal rim, a disc hemorrhage, or suspicious alteration in the nerve fiber layer), a visual field abnormality consistent with glaucoma, an elevated IOP greater than $21 \mathrm{~mm}$ Hg35. The diagnosis of a glaucoma suspect is also dependent on a normal open angle on gonioscopy [11]. Some authors have however classified glaucoma suspects into open angle and angle-closure suspects. The angleclosure suspects were based on the following criteria: posterior trabecular meshwork not visible 180 degrees, pigmented trabecular meshwork not visible 270 degrees without indentation or posterior trabecular meshwork not visible 180 degrees and IOP $22 \mathrm{mmHg}$ or greater. Studies conducted globally have used a wide variety of definitions to identify open-angle glaucoma suspects, reporting a prevalence of 1-8\% [12]. Optical coherence tomography (OCT), was introduced in 1991 as a new technique for high-resolution cross-sectional imaging of various ocular structures. The OCT was used in this study to assess the retinal nerve fiber layer thickness in glaucoma suspects attending the University of Port Harcourt Teaching Hospital (UPTH).

The retinal nerve fiber layer (RNFL) is formed by retinal ganglion cell axons and represents the innermost layer of the fundus. In this study $93.46 \mu \mathrm{m}$ is taken as the lower limit of normal average retinal nerve fiber layer thickness based on normative data gotten from normal northern Nigerian adults [13]. Normal nasal quadrant nerve fiber layer thickness $=85.10 \pm 23.60$.

\section{Methods}

This is a non-intervention observational hospital-based study using consecutive allocation of glaucoma suspects as they presented to the glaucoma clinic between October 2nd, 2012 and January 31st, 2013.The study population consisted of 120 glaucoma suspects who were seen at the outpatient clinic of the ophthalmology department between October 2012 and January 2013. Medical history was recorded and comprehensive ocular examination done on each of the subjects. Ocular examination included visual acuity, visual field, tonometry and ophthalmoscopy. Instruments used during the research were Pen torch for examination of the external structures of the eyes, Keeler ophthalmoscopes for fundus examination, Snellen's charts both literate and illiterate charts for visual acuity assessment, Reichert AT 555 Auto non-contact tonometer for measurement of the intra-ocular pressure. The anterior chamber angle was then examined with Goldmann three-mirror gonio-lens (Volks, indirect gonioscopy). Carl Zeiss Stratus OCT Model 3000 software version 4.0 was used to assess the retinal nerve fibre layer of the patients.
Approval to carry out this study was granted by the Ethical Committee of the University of Port Harcourt Teaching Hospital, Port Harcourt. The subjects included in the study were glaucoma suspects aged 18 years and above with open angles on gonioscopy (grade 3 and 4 Shaffers'system) consenting to the study. Those with normal central visual field and signal strength above 5 on optical coherence tomography testing were also included. The participant's pupils were then dilated using tropicamide $1 \%$ and phenylephrin 2.5\%. A slit lamp binocular indirect ophthalmoscopy using +78D (Volks) lens was used to examine the optic nerve head and retinal nerve fiber layer. Participants with superficial splinter haemorrages, focal loss of neuroretinal rim (notching), generalized loss of neuroretinal rim (VCDR $\geq 0.5$ ), cup-disc ratio asymmetry ( $\geq$ 0.2 ) or loss of retinal nerve fibers proceeded with the study. Also included were participants whose optic nerve head and nerve fibers appeared normal but had IOP greater than $21 \mathrm{mmHg}$. Redfree illumination of the posterior pole was also done to evaluate the retinal nerve fiber layer. A search for other abnormalities that may account for optic nerve changes such as; optic nerve pallor, disc drusen, optic nerve pits, disc edema due to central nervous system disease, macular degeneration, retinal vascular occlusion, and other retinal disease was also done. Automated visual-field examination was done using 24-2 Swedish interactive thresholding algorithm standard visual-field examination (Humphery visualfield analyzer, model 750). Participants with normal fields were then dilated for the OCT testing using tropicamide $1 \%$ and phenylephrine $2.5 \%$. After acquiring the best possible fixation and clear retinal video image, retinal nerve fiber layer ( RNFL) of each eye was imaged using fast-RNFL-thickness 3.4 scanning protocol, which automatically records three circular scans of diameter 3.4 $\mathrm{mm}$ around the center of the optic disc for 256 points along the scanning circle. Mean RNFL thickness in micrometers along the whole circle circumference, four quadrants, twelve clock hours, and at $256 \mathrm{~A}$-scan lengths were obtained. The sectors were defined in degrees, wherein $0^{\circ}$ was temporal horizontal point and the $360^{\circ}$ measurements along the circle were clockwise in right eye and anticlockwise in left eye. Superior quadrant was from $45^{\circ}$ to $135^{\circ}$, nasal from $135^{\circ}$ to $225^{\circ}$, inferior from $225^{\circ}$ to $315^{\circ}$, and temporal quadrant was from $315^{\circ}$ to $45^{\circ}$. Twelve $30^{\circ}$ sectors were also defined in clockwise order for right eye and in counterclockwise order for the left eye:

- $\quad$ Superior-nasal

- Nasal- superior

- Nasal

- Nasal- inferior

- Inferior- nasal

- Inferior

- Inferior-temporal

- Temporal- inferior

- Temporal 
- Temporal- superior

- $\quad$ Superior- temporal

- $\quad$ Superior

The same procedures for obtaining OCT measurements was followed for both eyes. Signal strength of 6 or higher is considered adequate for analysis of the results so scans with signal strengths below 6 were repeated and if the result was still poor, the participant was then excluded from the study. The data were analyzed using the statistical package EPI info version $6.04 \mathrm{~d}$, a software package designed by the Centers for Disease Control and Prevention (CDC), USA in 2001. Frequency was presented in percentages. Means and standard deviation were calculated for descriptive and comparative purposes. For comparison between the two groups, all data were subjected to student t-test and p-value.

\section{Results}

A total of 240 eyes of 120 participants were examined in this study. This was $100 \%$ coverage $\mathrm{Df}=6 \mathrm{MEAN} \pm \mathrm{SD}=42.8 \pm 13.79$ (Table 1).

Table 1: Age and sex distributions of study subjects.

\begin{tabular}{|c|c|c|c|c|c|}
\hline \multirow{2}{*}{ Age Group } & \multicolumn{2}{|c|}{ Sex } & \multirow{2}{*}{ Total\% } & \multirow{2}{*}{ Chi-Square } & \multirow{2}{*}{$P$ value } \\
\hline & Male\% & Female\% & & & \\
\hline$<20$ & $3(2.5)$ & $1(0.8)$ & $4(3.3)$ & 5.57 & $0.472^{*}$ \\
\hline $20-29$ & $7(5.8)$ & $14(11.7)$ & $21(17.5)$ & & \\
\hline 30-39 & $12(10.0)$ & $12(10.0)$ & $24(20.0)$ & & \\
\hline $40-49$ & 14 (11.7) & $17(14.2)$ & $31(25.8)$ & & \\
\hline 50-59 & $15(12.5)$ & $11(9.2)$ & $26(21.7)$ & & \\
\hline $60-69$ & $8(6.7)$ & $4(3.3)$ & $12(10.0)$ & & \\
\hline $70-79$ & $1(0.8)$ & $1(0.8)$ & $2(1.7)$ & & \\
\hline Total & $60(50.0)$ & $60(50.0)$ & $120(100.0)$ & & \\
\hline
\end{tabular}

\section{${ }^{*}$ Not Significant}

A total of $60(50 \%)$ males and $60(50 \%)$ females were examined giving a male to female ratio of 1:1 Table 1 . The ages range from 19 years to 75 years with a mean of $42.8 \pm 13.79$. The age group $40-49$ years had the highest representation $(25.8 \%$; $n=31 / 120)$ while those aged 70-79 years constituted the least $(1.7 \% ; n=2 / 120)$. There was no statistically significant difference in sex in gender representation ( $p>0.05)$ (Table 2$)$.

Table 2: RNFL Thickness in the different quadrants.

\begin{tabular}{|c|c|c|c|c|}
\hline RNFL & $\begin{array}{c}\text { Mean } \\
\text { Thickness }(\mu \mathrm{m})\end{array}$ & SD & t-test & p-value \\
\hline Inferior quadrant & 132.254 & \pm 24.772 & 23.9 & 0.001 \\
\hline Superior quadrant & 127.504 & \pm 26.455 & & \\
\hline Nasal quadrant & 80.533 & \pm 21.247 & & \\
\hline Temporal quadrant & 61.5 & \pm 16.980 & & \\
\hline
\end{tabular}

Table 3: Relationship between Age and RNFL thickness.

\begin{tabular}{|c|c|c|c|c|c|c|}
\hline Age Group & No. of eyes $(=240)$ & $\begin{array}{l}\text { Average RNFL } \\
\text { Thickness }\end{array}$ & $\begin{array}{c}\text { Inferior Quadrant } \\
\text { Fibers }\end{array}$ & $\begin{array}{c}\text { Superior } \\
\text { Quadrant Fibers }\end{array}$ & $\begin{array}{l}\text { Nasal Quadrant } \\
\text { Fibers }\end{array}$ & $\begin{array}{c}\text { Temporal } \\
\text { Quadrant Fibers }\end{array}$ \\
\hline In years & (freq) $(\%)$ & $($ Mean \pm SD $)$ & $($ Mean \pm SD $)$ & $($ Mean \pm SD $)$ & $($ Mean \pm SD $)$ & $($ Mean \pm SD) \\
\hline$<20$ & $8(3.3)$ & $105.65(12.14)$ & $133.75(16.86)$ & $135.13(27.53)$ & $89.50(21.03)$ & 64.75 (05.99) \\
\hline $20-29$ & $42(17.5)$ & $108.62(18.14)$ & $142.43(27.27)$ & $138.14(31.63)$ & $87.81(16.50)$ & $63.88(17.08)$ \\
\hline $30-39$ & $48(20.0)$ & $103.82(12.71)$ & 134.17 (19.47) & $134.44(20.98)$ & $80.48(19.21)$ & $65.75(21.68)$ \\
\hline $40-49$ & $62(25.8)$ & $102.99(15.69)$ & $134.87(25.45)$ & $129.94(26.74)$ & $82.65(20.02)$ & $63.66(14.60)$ \\
\hline $50-59$ & $52(21.7)$ & 92.60 (13.59) & $123.64(23.20)$ & 117.25 (21.39) & $75.86(27.27)$ & 54.69 (13.98) \\
\hline $60-69$ & $24(10.0)$ & 95.59 (10.55) & 131.75 (16.85) & $115.96(18.46)$ & $73.46(15.03)$ & $61.08(14.65)$ \\
\hline $70-79$ & $4(1.7)$ & $62.40(07.05)$ & $74.00(12.83)$ & $82.25(15.33)$ & $57.00(18.56)$ & $36.50(09.29)$ \\
\hline Total & $240(100.0)$ & & & & & \\
\hline t-test & & 3.26 & 2.65 & 2.6 & 1.73 & 1.99 \\
\hline P-value & & $0.001^{*}$ & $0.001^{*}$ & $0.001^{*}$ & $0.001^{*}$ & 0.001* \\
\hline Df & & 6 & 6 & 6 & 6 & 6 \\
\hline
\end{tabular}

*Not Significant

Table 2 shows the distribution of the RNFL in all the quadrants with the inferior quadrant having the thickest RNFL followed by the superior then the nasal with the temporal quadrant having the thinnest RNFL. This follows the 'I S N T' rule and was statistically significant with $\mathrm{p}<0.05$ (Table 3)
In Table 3,the age group 20-29 years had the thickest average retinal nerve fiber layer $(108.62 \pm 18.14)$ followed by those less than 20 years ( $105.65 \pm 12.14$ ) then in decreasing thickness 30-39years (103.82 \pm 12.71$), 40-49$ years(102.98 \pm 15.68$), 60-69$ years $(95.59 \pm 10.55), 50-59$ years $(92.60 \pm 13.59)$ and then $70-79$ years(62.40 \pm 7.05$)$. 
There is an obvious decrease of RNFL thickness with increasing age in all the quadrants and this is statistically significant with a $\mathrm{p}$-value $=0.001(\mathrm{p}<0.05)($ Figure 1$)$.
Figure 1 shows the Pearson correlation coefficient, $r$, is -0.38 . Because the value of ' $r$ ' is negative (-0.38), it implies that as age increases average RNFL is decreasing (Table 4).

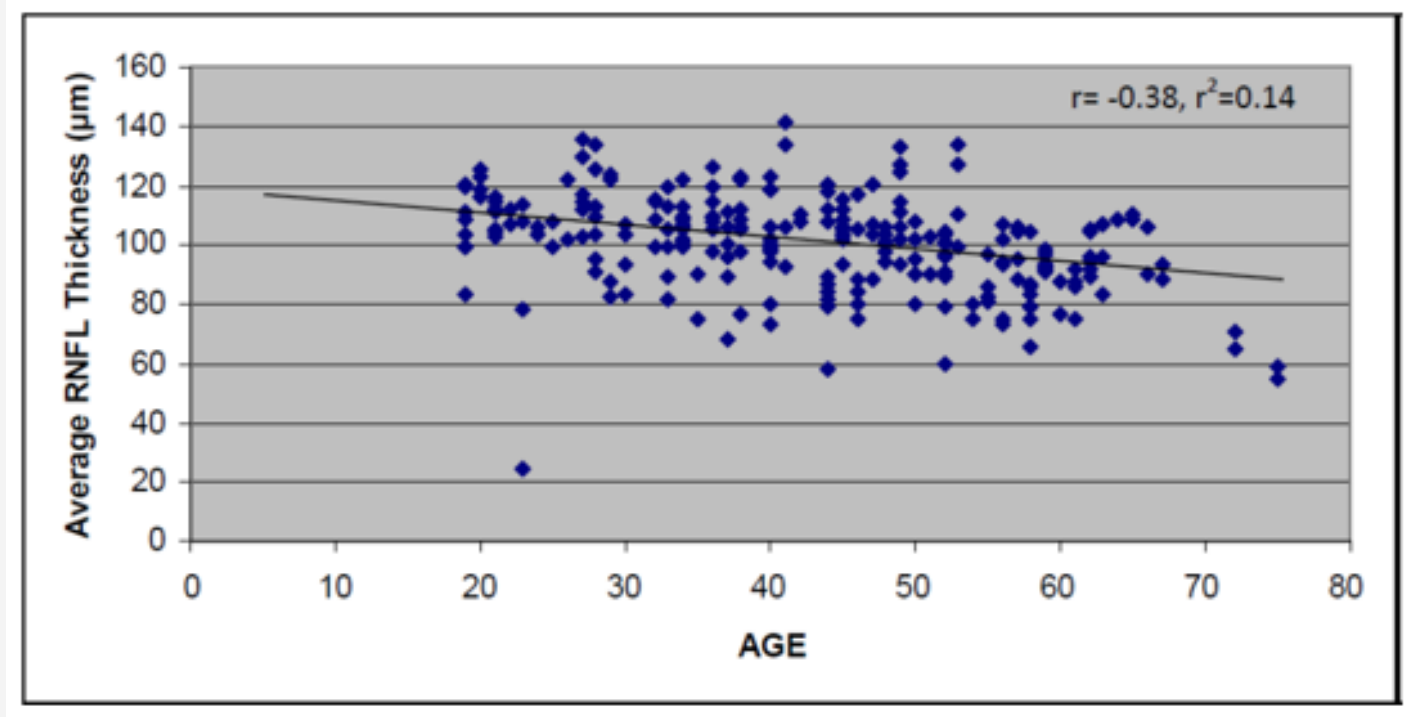

Figure 1:Scatter plot graph of age and average RNFL thickness.

Table 4: Correlation between age and RNFL thickness in the quadrants.

\begin{tabular}{|c|c|c|}
\hline \multirow{2}{*}{$\begin{array}{c}\text { RNFL Thickness in } \\
\text { the Quadrants }\end{array}$} & \multicolumn{2}{|c|}{ Age } \\
\cline { 2 - 3 } & Pearson Correlation Coefficient (r) & (r2) \\
\hline I Fibres & -0.26 & 0.07 \\
\hline S Fibres & -0.33 & 0.11 \\
\hline N Fibres & -0.23 & 0.05 \\
\hline T Fibres & -0.23 & 0.05 \\
\hline
\end{tabular}

Table 4 shows a weak negative correlation between age and the RNFL thickness in the four quadrants.

The proportion of eyes with abnormal RNFL thickness/thinning of RNFL

Most of the eyes $69.6 \%(n=167 / 240)$ had their average RNFL thickness greater than $93.46 \mu \mathrm{m}$

The remaining 30.4\% $(n=73 / 240)$ had their average RNFL thickness lesser than $93.46 \mu \mathrm{m}$

\section{Discussion}

The early diagnosis of glaucoma is a critical step in the management of the disease. If treated early, the prognosis for vision is excellent. One of the first structures to be damaged in glaucoma is the retinal nerve fiber layer (RNFL), which is the retinal layer containing the axons of the retinal ganglion cells. The ability to quantify changes in RNFL thickness early in the course of glaucoma is one of the features that make OCT appealing as a diagnostic instrument for glaucoma. This study provides information on the RNFL thickness of 240 eyes of 120 glaucoma suspects in the ophthalmology clinic of the University of Port Harcourt Teaching hospital (UPTH), and how it correlates to their age, disc diameter, vertical cup disc ratio (VCDR). A total of 120 participants, 60 $(50 \%)$ males and $60(50 \%)$ females were examined, this was not intentional as participants were consecutively recruited for the study. The age of participants examined ranged from 19 to 75 years with a mean age of $42.8 \pm 13.79$. The age group $40-49$ years had the highest population of those examined (31) while the age group 7079 constituted the least (2). There was no statistically significant difference in the age and sex distribution of the study sample.

The distribution of the peripapillary RNFL thickness in the various quadrants showed that the inferior quadrant had the thickest RNFL, followed by the superior, nasal and temporal quadrant. This was statistically significant and similar to the findings of in Northern Nigeria13, although their study population consisted of 190 healthy participants. This might imply that most of the glaucoma suspects examined in this study may actually be normal since many other studies have reported that the ISNT rule is violated in most confirmed glaucoma cases. In this study, a decrease of RNFL thickness is noted with increasing age in all the quadrants and this was statistically significant ( $p$-value $=0.001$ ) but the Pearson's correlation coefficient revealed a weak negative correlation( $r=-0.38, r 2=0.14)$ with only $14 \%$ of age values correlating with RNFL thickness of the participants examined. These findings are similar to the works done by Wong, et al. [14] in Hong Kong. Their study population had a mean age of $40 \pm 17$ and the age of participants correlated significantly with peripapillary RNFL thickness $(\mathrm{p}<0.001 ; \mathrm{r}=-0.28)$. In another study by Mok, et al. [15] in Hong Kong, there was a highly significant negative correlation in average RNFL measurement with increasing age (3.3 microns per decade, $\mathrm{p}<0.001)$. A significant negative correlation with increasing age was also identified in the RNFL measurements in all the four quadrants $(\mathrm{p}<0.05)$ and decreased at similar extent.

Similar works in other parts of the world have revealed that the RNFL thickness actually decreases with age [16]. The weak 
correlation in our study is probably due to the fact that some of the participants examined had early glaucoma with RNFL thinning despite their young age there by altering the normal correlation pattern. In this study the inferior and superior quadrant fibers ( $\mathrm{p}=0.001 ; \mathrm{r}=-0.26$ and $\mathrm{p}=0.001 ; \mathrm{r}=-0.33$ respectively) correlated with age more than the nasal $(p=0.001 ; r=-0.23)$ and temporal ( $p=0.001 ; r=-0.23$ ) quadrant fibers. Jong, et al. [17] also reported similar correlation in the superior and inferior quadrants in normal subjects. More studies will perhaps reveal if the nasal [1822 ] and temporal fibers can be used exclusively in the diagnosis of glaucoma since they relatively correlate poorly with age. The fact that RNFL thickness decreased significantly with advancing age should be borne in mind when interpreting OCT scans especially while monitoring glaucoma patients.

\section{Conclusion}

The distribution of the peripapillary RNFL thickness in the various quadrants showed that the inferior quadrant had the thickest RNFL, followed by the superior, nasal and temporal quadrant. The ISNT rule was obeyed in this study. A decrease of RNFL thickness is noted with increasing age in all the quadrants and this was statistically significant There is a weak negative correlation between age and the retinal nerve fiber layer thickness of glaucoma suspects therefore, age should be considered when analyzing optical coherence tomography print outs of glaucoma suspects. A total of $30.4 \%$ of the eyes had an average RNFL thickness lesser than $93.46 \mu \mathrm{m}$, which was the minimum normal value set for this study.

\section{Acknowledgement}

None.

\section{Conflicts of Interest}

No conflicts of interest.

\section{References}

1. George ACF, Jane D, Girkin CA, Gross RL, Peter A, et al. (2011) Introduction to Glaucoma: Terminology, Epidemiology and Heredity in Basic and clinical science course. American Academy of Ophthalmology, Singapore, pp. 3-7.

2. Buhrmann RR, Quigley HA, Barron Y, West SK, Oliva MS, et al. (2000) Prevalence of glaucoma in a rural East African population. Invest Ophthalmol Vis Sci 41(1): 40-48.

3. Ntim Amponsah CT, Amoaku WMK, Ofosu-Amaah S, Ewusi RK; Idirisuriya Khair R, et al (2004) Prevalence of glaucoma in an African population. Eye 18(5): 491-497.

4. Adeyinka Ashaye (2010) Glaucoma-Global Facts: in GLAUCOMA BLINDNESS facts, fancies and fables. Annual Faculty Lecture Ibadan, Book builders, Nigeria, pp. 6-7.
5. (2012) National population commission, Nigeria.

6. Rabiu MM, Kyari F, Ezelum C, Elhassan E, Sanda S, et al. (2012) Review of the publications of the Nigeria national blindness survey: Methodology, prevalence, causes of blindness and visual impairment and outcome of cataract surgery. Ann Afr Med 11(3): 125-130.

7. Awoyesuku EA, Ejimadu CS (2012) Visual disability in newly diagnosed Primary open Angle Glaucoma (POAG) patients in a tertiary Hospital in Nigeria. Niger J Med 21(1): 78-80.

8. Omoni AO (2005) The Epidemiology of blindness and visual impairment in a fishing village in Nigeria. The Nigerian Health Journal 15(1,2): 252260.

9. Pedro Egbe CN (2006) Blindness and visual impairment in the NigerDelta: a study of Ahoada East LGA of Rivers State Nigeria. P Med J 1(1): 56-61.

10. Pedro Egbe CN, Chukwuka IO (2010) Subjective Optic Disc Assessment and Single Measurement Intraocular Pressure to Screen a Cohort of Pensioners in Port Harcourt. Nigerian Journal of Ophthalmology 18: 6265.

11. George ACF, Jane D, Girkin CA, Gross RL, Peter A, et al. (2011) Open-Angle Glaucoma: in: Basic and Clinical Science Course, American Academy of Ophthalmology, Singapore, pp. 96-99.

12. Yanoff M, Duker JS (2008) Epidemiology of Glaucoma. Mosby (ed.) In: Ophthalmology ( $3^{\text {rd }}$ Edn), Spain.

13. Sani RY, Abdu L, Pam V (2012) Retinal Nerve Fibre Layer Thickness in normal Northern Nigerian adults using Optical coherence tomography. Presentation at the OSN Scientific conference.

14. Wong YH, Wong CM, Chan WN (2010) Relationship between age and peripapillary retinal nerve fibre layer thickness: an optical coherence tomography study. Hong Kong Med J 16: 265-268.

15. Mok KH, Lee VW, So KF (2002) Retinal nerve fiber layer measurement of the Hong Kong Chinese population by optical coherence tomography. J Glaucoma 11(6): 481-483.

16. Balazsi AG, Rootman J, Drance SM, Schulzer M, Douglas GR (1984) The effect of age on the nerve fiber population of the human optic nerve. Am J Ophthalmol 97: 760-766.

17. Jong YL, Young HH, Sun ML, Yong YK (2012) Age and Retinal Nerve Fiber Layer Thickness Measured by Spectral Domain Optical Coherence Tomography. Korean J Ophthalmol 26(3): 163-168.

18. Jonas JB, Schmidt AM, Muller Bergh JA, Schlötzer-Schrehardt UM, Naumann GO (1992) Human optic nerve fiber count and optic disc size. Invest Ophthalmol Vis Sci 33(6): 2012-2018.

19. Schuman JS, Pedut Kloizman T, Hertzmark E, Hee MR, Wilkins JR (1996) Reproducibility of nerve fiber layer thickness measurements using optical coherence tomography. Ophthalmology 103(11): 1889-1898.

20. Jonas JB, Gusek GC, Naumann GO (1988) Optic disc, cup and neuroretinal rim size, configuration and correlations in normal eyes. Invest Ophthalmol Vis Sci 29(7): 1151-1158.

21. Racette L, Boden C, Kleinhandler SL, Girkin CA, Liebmann JM, et al. (2005) Differences in visual function and optic nerve structure between healthy eyes of blacks and whites. Arch Ophthalmol 123(11):1547-1553.

22. Teal PK, Morin JD, McCulloch C (1972) Assessment of the normal disc. Trans Am Ophthalmol Soc 70:164-177. 